\title{
The ZEPLIN-III dark matter detector: Instrument design, manufacture and commissioning
}

D.Yu. Akimov ${ }^{\text {a }}$, G.J. Alner ${ }^{\mathrm{b}}$, H.M. Araújo c,b ${ }^{\mathrm{c}}$, A. Bewick ${ }^{\mathrm{c}}$, C. Bungau ${ }^{\mathrm{c}, \mathrm{b}}$, A.A. Burenkov ${ }^{\mathrm{a}}$, M.J. Carson ${ }^{\mathrm{d}}$, H. Chagani ${ }^{\mathrm{d}}$, V. Chepel ${ }^{\mathrm{e}}$, D. Cline ${ }^{\mathrm{f}}$, D. Davidge ${ }^{\mathrm{c}}$, E. Daw ${ }^{\mathrm{d}}$, J. Dawson ${ }^{\mathrm{c}}$, T. Durkin ${ }^{\mathrm{b}}$, B. Edwards ${ }^{\mathrm{c}, \mathrm{b}}$, T. Gamble ${ }^{\mathrm{d}}$, C. Chag ${ }^{\mathrm{g}}$, R.J. Hollingworth ${ }^{\mathrm{d}}$, A.S. Howard ${ }^{\mathrm{c}}$, W.G. Jones ${ }^{\text {c }}$, M. Joshi ${ }^{c}$, K. Mavrokoridis ${ }^{d}$, E. Korolkova ${ }^{g}$, A. Kovalenko ${ }^{\text {a }}$, V.A. Kudryavtsev ${ }^{d}$, I.S. Kuznetsov ${ }^{\text {a }}$, T. Lawson ${ }^{\mathrm{d}}$, V.N. Lebedenko ${ }^{\mathrm{c}}$, J.D. Lewin ${ }^{\mathrm{b}}$, P. Lightfoot ${ }^{\mathrm{d}}$, A. Lindote ${ }^{\mathrm{e}}$, I. Liubarsky ${ }^{\mathrm{c}}$, M.I. Lopes ${ }^{\mathrm{e}}$, R. Lüscher ${ }^{\mathrm{b}}$, J.E. McMillan ${ }^{\text {d, P. Majewski }}{ }^{\text {, }}$, B. Morgan ${ }^{\mathrm{d}}$, D. Muna ${ }^{\mathrm{d}}$, A.S. Murphy ${ }^{\mathrm{g}}$, F. Neves ${ }^{\mathrm{e}}$, G.G. Nicklin ${ }^{\mathrm{d}}$, S.M. Paling ${ }^{\mathrm{d}}$, D. Muna ${ }^{\mathrm{d}}$, J. Pinto da Cunha ${ }^{\mathrm{e}}$, S.J.S. Plank ${ }^{\mathrm{g}}$, R. Preece ${ }^{\mathrm{b}}$, J.J. Quenby ${ }^{c}$, M. Robinson ${ }^{\mathrm{d}}$, C. Silva ${ }^{\mathrm{e}}$, V.N. Solovov ${ }^{\mathrm{e}}$, N.J.T. Smith ${ }^{\mathrm{b}}$, P.F. Smith ${ }^{\mathrm{b}}$, N.J.C. Spooner ${ }^{\text {d }}$, V. Stekhanov ${ }^{a}$, T.J. Sumner ${ }^{c}, *$, C. Thorne ${ }^{c}$, D.R. Tovey ${ }^{d}$, E. Tziaferi ${ }^{d}$, R.J. Walker ${ }^{c}$, H. Wang ${ }^{\text {f }}$, J. White ${ }^{\text {h }}$, F. Wolfs ${ }^{\text {i }}$

\author{
${ }^{a}$ Institute for Theoretical and Experimental Physics, Moscow, Russia \\ ${ }^{\mathrm{b}}$ Particle Physics Department, Rutherford Appleton Laboratory, Chilton, UK \\ c Astrophysics Group, Blackett Laboratory, Imperial College London, London SW7 2BZ, UK \\ ${ }^{\mathrm{d}}$ Physics and Astronomy Department, University of Sheffield, UK \\ ${ }^{\mathrm{e}}$ LIP-Coimbra and Department of Physics of the University of Coimbra, Portugal \\ ${ }^{\mathrm{f}}$ Department of Physics and Astronomy, University of California, Los Angeles, USA \\ ${ }^{\mathrm{g}}$ School of Physics, University of Edinburgh, UK \\ ${ }^{\mathrm{h}}$ Texas A\&M University, USA \\ ${ }^{\mathrm{i}}$ University of Rochester, New York, USA
}

Received 12 May 2006; received in revised form 4 September 2006; accepted 5 September 2006 Available online 12 October 2006

\begin{abstract}
We present details of the technical design, manufacture and testing of the ZEPLIN-III dark matter experiment. ZEPLIN-III is a twophase xenon detector which measures both the scintillation light and the ionisation charge generated in the liquid by interacting particles and radiation. The instrument design is driven by both the physics requirements and by the technology requirements surrounding the use of liquid xenon. These include considerations of key performance parameters, such as the efficiency of scintillation light collection, restrictions placed on the use of materials to control the inherent radioactivity levels, attainment of high vacuum levels and chemical contamination control. The successful solution has involved a number of novel design and manufacturing features which will be of specific use to future generations of direct dark matter search experiments as they struggle with similar and progressively more demanding requirements. (C) 2006 Elsevier B.V. All rights reserved.
\end{abstract}

Keywords: ZEPLIN-III; Dark matter; Liquid xenon; Radiation detectors; WIMPs

\footnotetext{
* Corresponding author.

E-mail address: t.sumner@imperial.ac.uk (T.J. Sumner).
} 


\section{Introduction}

ZEPLIN-III is a two-phase (liquid/gas) xenon detector developed and built by the ZEPLIN Collaboration, ${ }^{1}$ which will try to identify and measure galactic dark matter in the form of weakly interacting massive particles, or WIMPs $[1,2]$. Upon completion of physics testing now underway at Imperial College, the system will join the ZEPLIN-II [3] and DRIFT-IIa [4] experiments already operating $1100 \mathrm{~m}$ underground in our laboratory at the Boulby mine (North Yorkshire, UK).

Two-phase emission detectors based on the noble gases date back several decades [5]. In the last decade, this technology has gained a new momentum in view of increasing interest for searching rare events, WIMPs in particular, requiring both large detection masses and high discrimination against background. In its previous work, the ZEPLIN Collaboration has explored the potential of high-field xenon systems to enhance sensitivity and background discrimination [6-8]. The operating principle relies on different particle species generating different amounts of vacuum ultraviolet (VUV) scintillation light and ionisation charge in liquid xenon (LXe). The ratio between these two signal channels provides a powerful technique to discriminate between electron and nuclear recoil interactions. WIMPs are expected to scatter elastically off Xe atoms, much like neutrons, and the recoiling nucleus will produce a different signature to $\gamma$-ray interactions and other sources of electron recoils.

WIMP detectors differ from more traditional detectors of nuclear radiation in that they require: (i) extremely low radioactive and cosmic-ray backgrounds, addressed by the use of radio-pure materials and operation deep underground; (ii) excellent discrimination of the remaining background events, especially for electron recoils; (iii) a low-energy threshold for nuclear recoils, since the kinematics of WIMP-nucleus scattering results in a very soft recoil spectrum $(\lesssim 100 \mathrm{keV})$.

Monte Carlo simulations [9,10] were essential in key areas to inform the design of the ZEPLIN-III instrument. Acceptable levels of trace contamination must be set for all detector materials, requiring simulations of internal and external backgrounds expected from each component. Cosmic-ray-induced backgrounds also need careful calculation, since experimental measurements would require nothing short of a dedicated WIMP detector. These simulations establish the residual electron/photon and neutron event rates and spectra. Detailed detector simulations leading to predicted data time-lines were used to find the level of discrimination and energy threshold which can realistically be achieved. Feedback from this process into the design process has been essential. In addition, the data produced by two-phase detectors are often complex, and par-

\footnotetext{
${ }^{1}$ Edinburgh University, Imperial College London, ITEP-Moscow, LIPCoimbra, Rochester University, CCLRC Rutherford Appleton Laboratory, Sheffield University, Texas A\&M, UCLA.
}

ticular simulations are required to help extract actual physics parameters. Finally, realistic datasets help with planning the data acquisition electronics and the data analysis software.

In this paper we describe the instrument design philosophy, the engineering design solutions and the manufacturing processes adopted. We also provide first test results obtained in a surface laboratory. In a separate paper [11] we present full performance Monte Carlo simulations for the final, as built, instrument.

\section{The ZEPLIN-III instrument}

There are four important design requirements for a dark matter detector: a low-energy threshold, good particle discrimination, 3-D position reconstruction and a low background within the fiducial volume. The ZEPLIN-III approach, as shown at a target concept level in Fig. 1 and at a detailed system engineering level in Fig. 2, tries to push the boundaries of the two-phase xenon technique to simultaneously achieve the best performance possible in these four aspects.

ZEPLIN-III achieves a low threshold for the primary scintillation by placing its photodetectors, photomultipliers (PMTs), in the liquid phase and by using a flat planar geometry. Using PMTs in the liquid removes two interfaces, both with large refractive index mismatches and puts in an additional interface at which total internal reflection also works to improve the light collection for the primary scintillation. The planar geometry gives a large solid angle acceptance and lessens the dependance on surface reflectivities. A low threshold for the electroluminescence from the gas phase which provides the secondary signal is achieved by using a high electric field in the gas region to produce high levels of photon emission per electron emitted from the surface and by using refraction at the liquid surface to produce a 'focusing' effect for the light onto the immersed PMT array.

Good particle discrimination between the nuclear recoil signals expected from WIMPs and the electron recoils from photon backgrounds comes from employing a two-phase design which allows both scintillation and ionisation to be measured for each event. The scintillation output (signal $\mathrm{S} 1$ in Fig. 1) is a prompt, fast signal (time-constant of a few $10 \mathrm{~s}$ of $\mathrm{ns}$ ) from an interaction in the liquid phase. Any ionisation escaping from the interaction site is drifted by an applied electric field towards the liquid surface. In ZEPLIN-III the electric field is high enough that the electrons are extracted into the gas phase where they then cause an extended proportional electroluminescence light output (signal S2 in Fig. 1) as they drift across the gas gap. The S1 and S2 signals are separated in time by an amount corresponding to the drift distance in the liquid phase (up to $17 \mu$ s for the $3.5 \mathrm{~cm}$ depth) and so both signals can be readout by the same PMTs. The ratio of S2 to S1 depends on the particle species. The effectiveness of this discrimination depends on the width and separation of the 

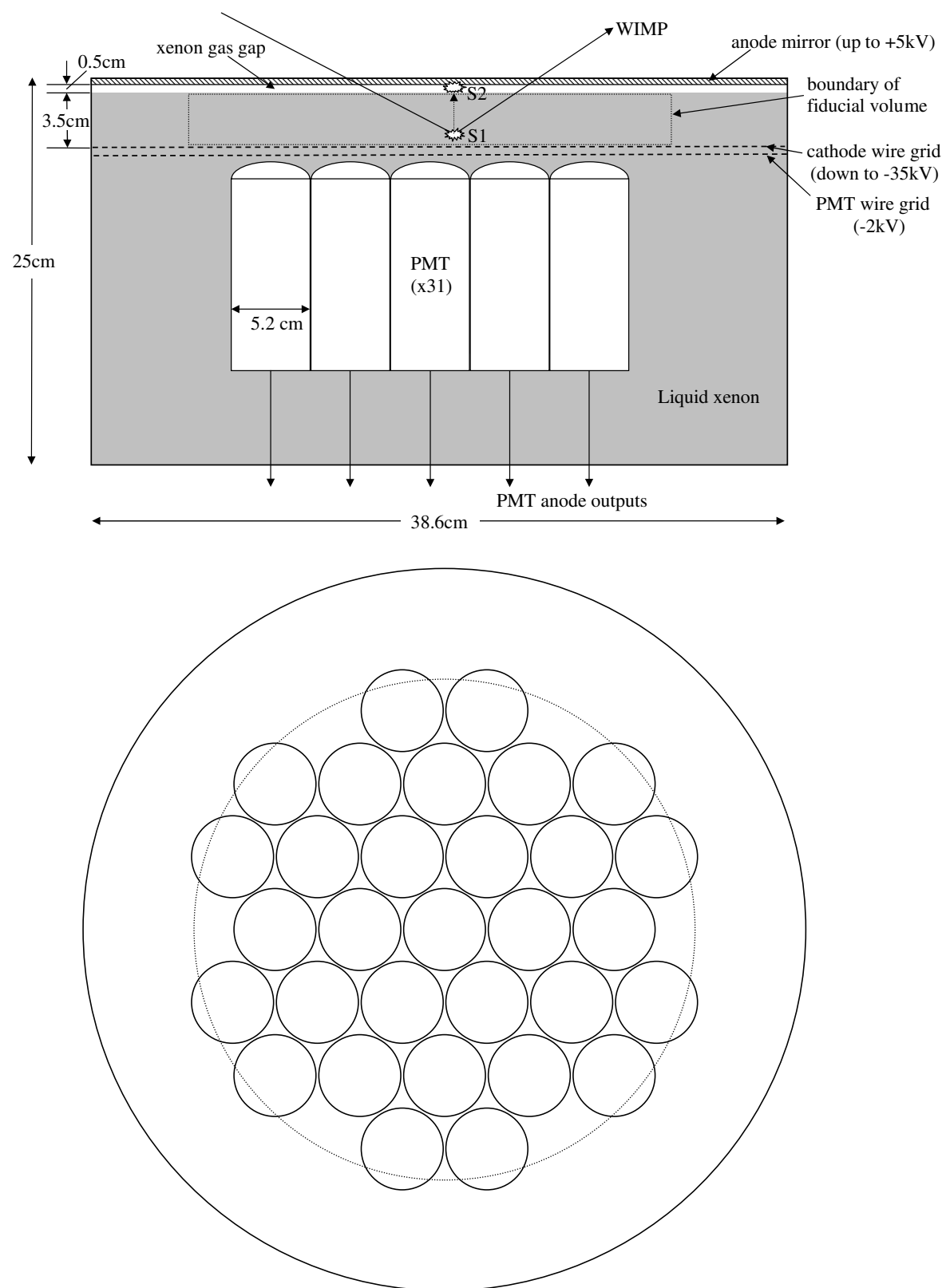

Fig. 1. Cross-sections of the target volume showing the key system design concepts and the event interaction process. The top panel shows a side view with key design features labeled, including a boundary box for the fiducial volume. The bottom panel provides a top view of the PMT arrangement and the radial fiducial boundary.

distributions for each species. It turns out [12] that the discrimination is improved by working at moderate electric fields which increases the separation between the two distributions and improves the statistical uncertainties of the ionisation signal. Some discrimination against nuclear recoil signals from neutron elastic scattering is obtained by having good 3-D position reconstruction which can identify the multiple scattering expected from the much higher cross-sections for neutron scattering than for WIMP scattering. Efficient measurement of the ionisation relies on achieving a long lifetime against trapping for free electrons in the liquid. This requires ultrapure xenon as free from electronegative impurities as possible. The target volumes must be constructed as high vacuum vessels and a dedicated gas purification system is needed.

ZEPLIN-III achieves good 3-D position reconstruction by using an array of $312^{\prime \prime}$ diameter photomultipliers as shown in the lower panel in Fig. 1. The pattern of signals seen in the PMTs can provide sub-cm 2-D spatial resolution in the horizontal $r, \theta$ plane even for single electrons extracted from the liquid [34]. Resolution in the $z$ co-ordinate at the $\sim 50 \mu \mathrm{m}$ level is obtained from the time interval between the S1 and S2 signals. The 3-D position reconstruction is then used to define the fiducial volume without reliance on any physical surfaces. As shown in [11] this allows a fiducial region of diameter $31.2 \mathrm{~cm}$ containing 


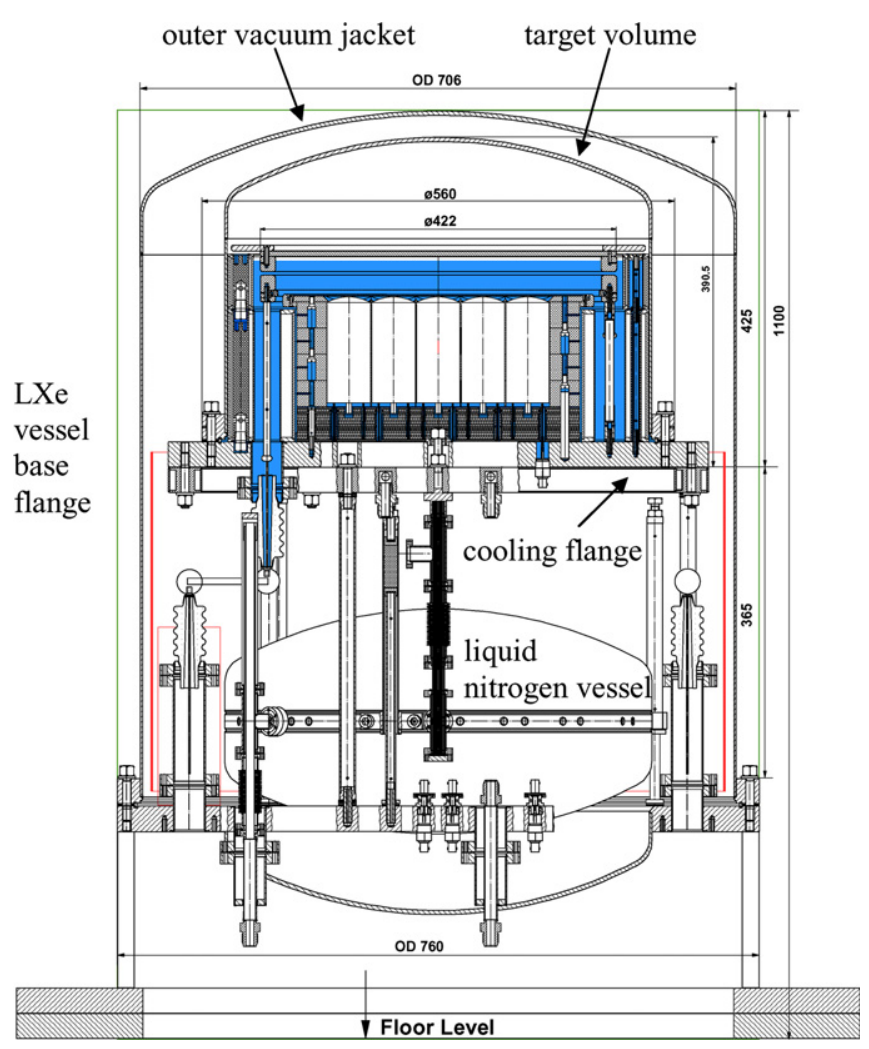

Fig. 2. Cross-sectional views of the complete ZEPLIN-III instrument showing the key sub-system components. Scale dimensions are in $\mathrm{mm}$ and ZEPLIN-III stands $1100 \mathrm{~mm}$ tall with a diameter of $760 \mathrm{~mm}$. The bulk of the parts are made of $\mathrm{C} 103$ OFHC copper.

$8 \mathrm{~kg}$ of xenon. The boundaries of this volume are shown as the dotted lines in Fig. 1.

Low background is achieved partly by operation underground and partly by using a very restricted range of materials for its construction. Although the PMTs are the largest specific contributors to the background budget it is important that careful attention is paid to all materials used as the masses of these can exceed the PMT mass by up to two orders of magnitude. The PMTs were made from materials which were all screened in our underground laboratory prior to manufacture. Hence we have a wellfounded measure of their activity: the measured levels were $250 \mathrm{ppb}$ in U, $290 \mathrm{ppb}$ Th and $1350 \mathrm{ppm}$ in K. From our Monte Carlo simulations this gives a background of 10 events $/ \mathrm{kg} / \mathrm{day} / \mathrm{keV}$ in the low-energy region. It is planned to eventually replace these PMTs by low-background versions which are currently in development with a 30 -fold reduction in their activity.

In the following sections we detail the design and manufacture of the individual parts of the ZEPLIN-III experiment. These include the target volume, the cooling system, the outer vacuum jacket, the gas handling system, including the safety reservoirs, and the data acquisition system. In the final section we provide data from surface commissioning tests which validate the key performance parameters for ZEPLIN-III.

\section{The target volume}

The detailed design of the inner components within the target volume is shown in Fig. 2.

\subsection{The PMT array}

Inside the xenon vessel is the array of 31 PMTs, immersed in the liquid phase, looking up to a $\simeq 40-\mathrm{mm}$ thick liquid xenon layer on top of which is a $5-\mathrm{mm}$ xenon gas gap. The figure shows a cross-sectional view through a centre line passing through 5 of the $52-\mathrm{mm}$ diameter PMTs. The others are arranged in a hexagonal closepacked array with a pitch spacing of $54 \mathrm{~mm}$. A pure copper 'screen' has an array of 53-mm holes into which the PMTs fit. This provides both light screening and electrical isolation between the PMTs. It has an outer diameter of $340 \mathrm{~mm}$ and a height of $128 \mathrm{~mm}$. For ease of manufacture the total height of the 'screen' was made in four sections. Each PMT hole through the copper 'screen' has a diameter of $53 \mathrm{~mm}$ giving a $1-\mathrm{mm}$ wall minimum thickness between each PMT. Two techniques were used to produce such thin wall section through such a thickness of copper; wire erosion and boring. Both worked but the boring produced a better surface finish. Sitting directly on top of the 'screens' is another copper disc with holes in it. This time the thickness is $7 \mathrm{~mm}$ and the holes are finished with highly polished conical sections to improve the light collection; this plate is hence referred to as the 'PMT mirror'.

Each PMT has 15 pins to which connections must be made (12 dynodes, anode, cathode and focus). However it would be impractical to bring all 465 connections out through individual UHV electrical feeds through the bottom thick copper flange. Instead all the PMTs are run from a common high voltage supply and dynode distribution system which reduces the amount of feedthroughs to just 47. The corresponding dynode pins on each PMT are connected together using a stack of 16 thin copper plates, held apart with small quartz spacers, below the PMT array. Each 2-mm thick plate has a different pattern of holes (see Fig. 3) allowing connection to each pin in turn whilst the others pass through with clearance. Connections between the copper plates and the PMT contacts were done by first cold welding a pin into the copper plate and then using spring-loaded tubes to join the two pins together (see Fig. 4). The pins used in the copper plates were made in copper with a gold coating and these were inserted into tight fitting holes in the plates using a drill press. The spring contacts were made from stainless-steel tubing with reduced wall sections and slots. These contacts provide enough friction for retention of the PMT against buoyancy forces during immersion in liquid xenon. Connection between each plate and its single UHV coaxial feedthrough was again made by a direct spring-loaded tube but with the addition of gold-plated copper wires with silver-plated copper adaptors to provide the extensions between end contacts. The anode connection from each PMT is brought 


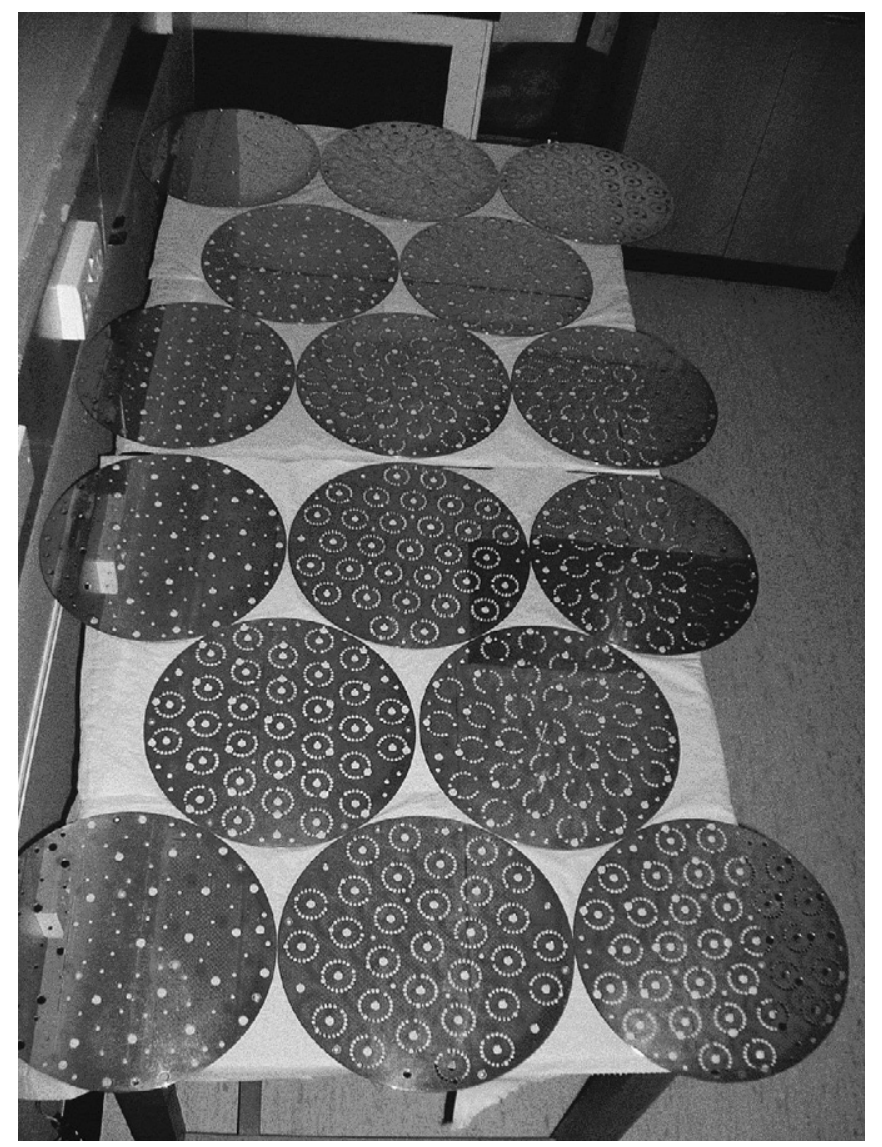

Fig. 3. The $162-\mathrm{mm}$ copper plates used to make the internal PMT dynode interconnections.

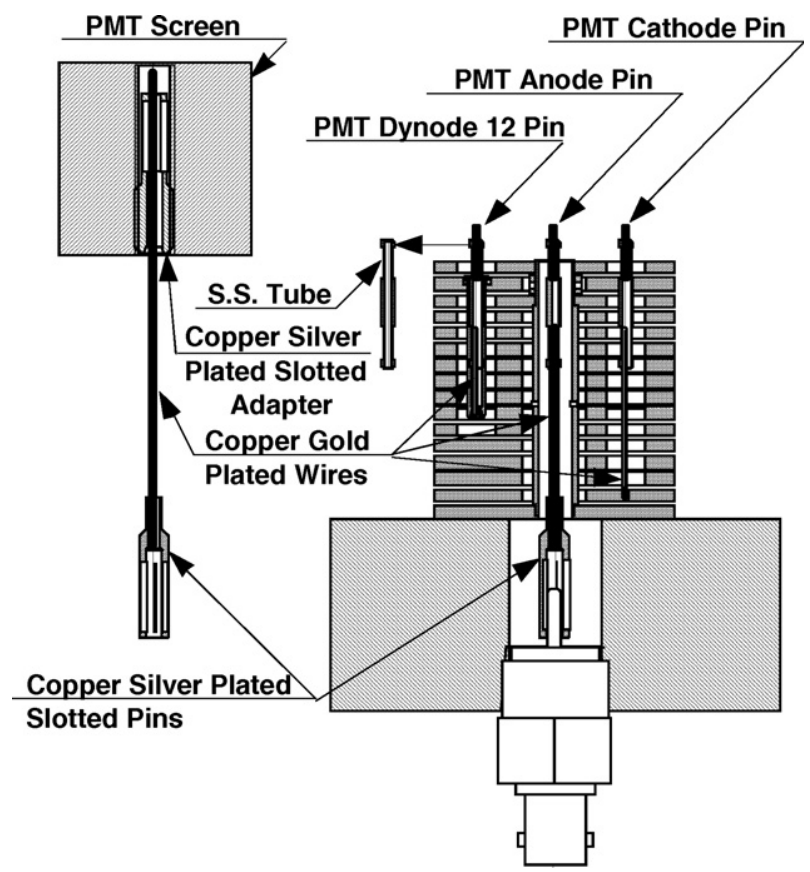

Fig. 4. Various spring-loaded contacts used to connect the PMT pins to the copper plates.

out separately on a dedicated coaxial UHV feedthrough in a similar way. The specific arrangement of the 16 copper plates can be seen in Fig. 4. The upper and lower plates are connected to ground. The second lowest plate is connected to the PMT cathodes, the next 11 are connected to dynodes 1-11 in turn. Above that there is then another grounded plate and between this and the top ground plate is dynode 12. The two grounded plates either side of the dynode 12 plate deliberately provide both extra capacitance to ground for that dynode and prevent cross-coupling with other connections. Copper tubes provide shielding along the run of each anode output connection. Shielded cables pass across the outer vacuum jacket space to connectors in its base plate. A single external voltage divider chain is used to provide all the common dynode voltages. To ensure reasonably well matched gains when running from a common HV supply, PMTs were procured with gains within prescribed limits. Once selected the batch of 35 PMTs (ETL D730/9829Q) was tested and calibrated at low temperature with Xe scintillation UV light prior to installation in the detector [13]. The PMTs were customised specifically for ZEPLIN-III in two ways: firstly a conductive pattern of so-called 'fingers' was deposited on the inside of the window to avoid saturation at high count rates, and secondly to provide a modified pin-out arrangement to facilitate the use of the copper interconnection plates. The PMTs are operated with the anode at ground potential.

\subsection{The electric field}

Proper operation in two-phase mode requires that there be a sufficiently high and constant electric field in three distinct regions. In the active volume of the detector the electric field helps to separate ionisation charge released from the track of the interacting particle before it can recombine. This field must be directed such that the electrons start 'drifting' towards the liquid surface. Hence the field in this first region is called the 'drift' field. The second critical region is at the liquid/gas interface. Here the field in the liquid must be high enough to efficiently extract the electrons into the gas phase. This not only increases the signal strength but also prevents charge build-up at the surface. This field is called the 'extraction' field. Finally in the gas phase the field must be high enough for the accelerated electrons to produce excitation in the gas atoms. The excited atoms then form excited dimers followed by dissociative radiative emission in the usual way, which produces the signal seen by the PMTs. This last field is called the 'electroluminescence' field. These three fields can either be produced by setting up a segmented electrode structure producing distinct regions, as is done in ZEPLIN-II [14], or, as in the case of ZEPLIN-III, a single pair of outer electrodes can be used to produce all three at once. The advantage of the latter is the absence of any physical electrode structure in the liquid which could then be a source of background and/or feedback. However it does mean that a single much higher individual voltage is required and the fields cannot be controlled independently. The two 
electrodes used are the solid flat plate ('anode mirror') above the gas gap and a wire plane ('cathode grid') $40 \mathrm{~mm}$ below it in the liquid. The 8-mm top plate is made from copper and its bottom surface has been lapped using optical techniques and left highly polished. Up to $40 \mathrm{kV}$ can be applied between the two 'electrodes'.

A second wire grid ('PMT grid') is located $5 \mathrm{~mm}$ below the cathode grid and just above the PMT array. This defines a reverse field region just above the PMTs which suppresses secondary signals from low-energy background photons from the PMTs and also helps isolate the internal PMT photocathode fields from the external high electric field. The diameter of the electrode structure is $38.6 \mathrm{~cm}$, whilst that of the PMT array is $34 \mathrm{~cm}$. The fiducial volume will be defined by a combination of primary to secondary timing and radial position recovery from the PMT hit pattern. The timing cut will confine the fiducial volume in depth to exclude regions close to the cathode grid and the liquid surface. The radial position recovery from the PMT hit pattern will be used to confine the radial extent of the fiducial volume to well inside the PMT array diameter. These two cuts define a region in which the electric field is most uniform. This is particularly important for charge collection to reduce any position dependence on either charge removal from the interaction site and/or transport efficiency into the gas phase. A field simulation produced using ANSYS [15], is shown in Fig. 5. There is no solid or conducting field shaping structure between the anode mirror and cathode grid except at the very rim. This is a deliberate design feature which avoids any 'surface' effects causing potential problems, from surface trapping, embedded radioactivity or photoelectric feedback. At the rim the electrodes are brought close together which helps prevent any electrons released from those surfaces below the liquid level from escaping into the gas phase.
The electric fields in the liquid and gas phases, $E_{1}$ and $E_{\mathrm{g}}$, within the radius of the fiducial region are thus well approximated by parallel plate formulae:

$E_{1}=\frac{\Delta V}{\epsilon_{\mathrm{r}} L-\left(\epsilon_{\mathrm{r}}-1\right) D}$

$E_{\mathrm{g}}=\epsilon_{\mathrm{r}} E_{1}$

where $\epsilon_{\mathrm{r}}$ is the relative permittivity of liquid xenon, $\Delta V$ is the voltage difference applied between the anode mirror and the cathode grid, $L$ is the distance between the mirror and grid and $D$ is the liquid depth above the grid.

Over the fiducial volume the uniformity in $E_{1}$ will be limited by variations in $L$ and $D$ according to

$\frac{\Delta E_{1}}{E_{1}}=\frac{\epsilon_{\mathrm{r}} \Delta L+\left(\epsilon_{\mathrm{r}}-1\right) \Delta D}{\epsilon_{\mathrm{r}} L-\left(\epsilon_{\mathrm{r}}-1\right) D}$

$\Delta L$ will have three components: planarity of the anode mirror, planarity of the cathode grid, and parallelism of the two. The anode mirror has been lapped using optical polishing techniques. The cathode grid wire is positioned using precision placement slots in the formers and have sufficient pre-tension to limit displacement due to electrostatic forces when the voltages are applied. The parallelism of the two planes is determined by manufacturing tolerances and assembly checks. The combination of these is $\Delta L \sim$ $\pm 100 \mu \mathrm{m}$. $\Delta D$ depends on degree of parallelism of the liquid surface with the two electrode planes. An active control system allows leveling of the instrument based on capacitive measurements at three locations around the circumference. As is shown later these have sub-mm resolution which translates to $\Delta D \sim \pm 500 \mu \mathrm{m}$ within the fiducial radius. Using values of $\epsilon_{\mathrm{r}}=2, L=40 \mathrm{~mm}$ and $D=35 \mathrm{~mm}$, then gives $\Delta E_{1} / E_{1}<15 \%$. At our maximum operating voltage difference of $40 \mathrm{kV}$ the electric field in

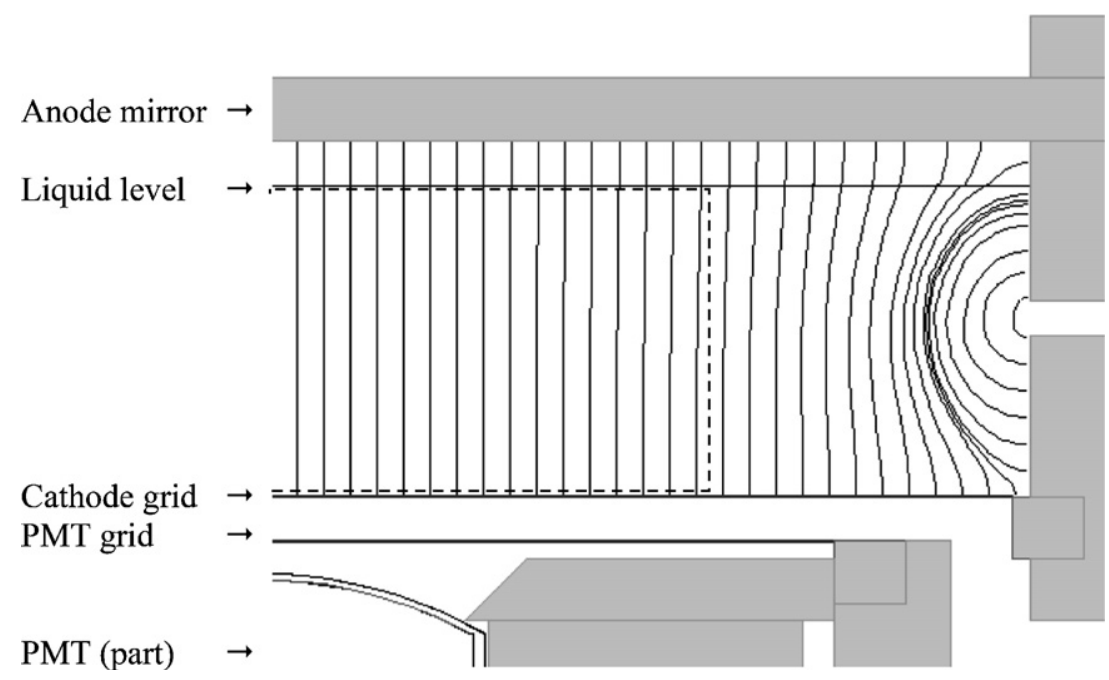

Fig. 5. Electron trajectories close to the field shaping electrodes, showing the high level of uniformity reached at the fiducial volume boundary. The computations were done using ANSYS [15]. The dashed line shows the boundary of the fiducial volume, which is kept well away from the region where the drift-paths become distorted. 

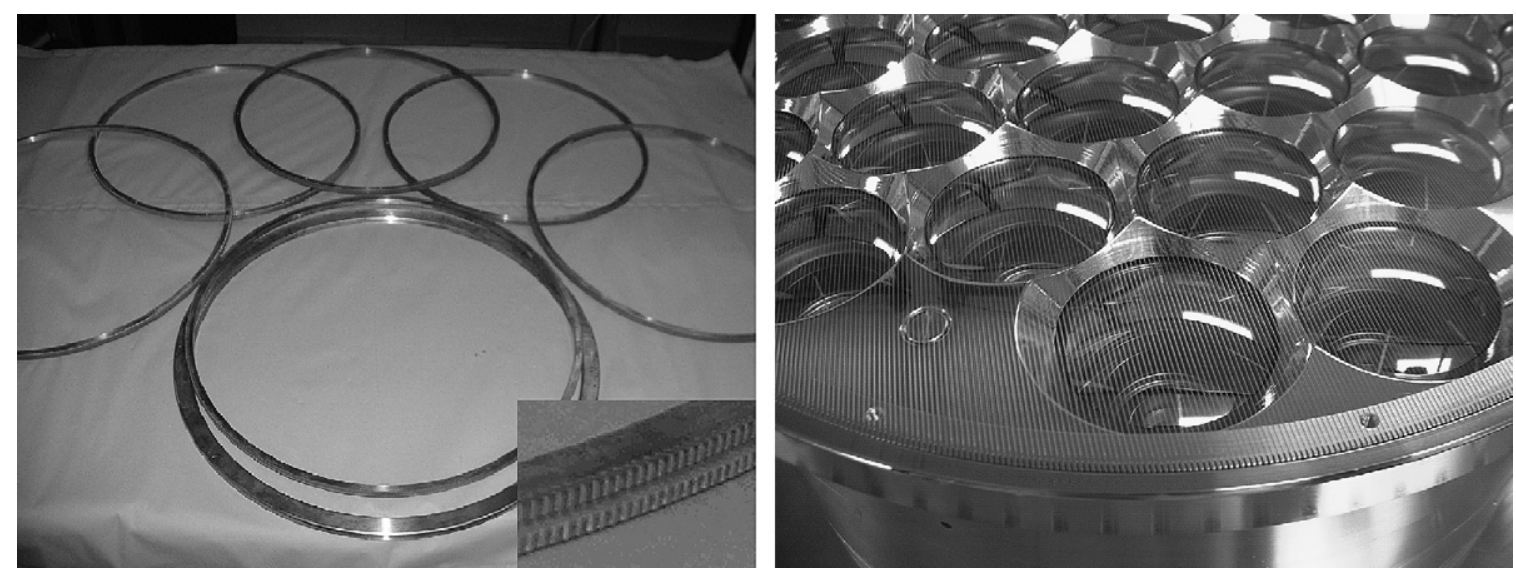

Fig. 6. On the left are the copper formers for the wire grids. The inset detail shows an expanded view in which the slots cut to control to wire positioning can be seen. On the right is a view of the assembled PMT array in which the PMT grid can be seen.

the liquid is $8.9 \mathrm{kV} / \mathrm{cm}$ and this may vary by up to $1.3 \mathrm{kV} /$ $\mathrm{cm}$ within the fiducial volume.

The variation in electric field will affect the primary and secondary signal amplitudes and the time delay between them. Only the effect on the secondary amplitude is of any consequence as the other two are slowly-varying functions of the electric field at our operation point [16-18]. For example, the primary scintillation amplitude is only affected at the $\lesssim 1 \%$ level, which is negligible compared to intrinsic fluctuations due to photon/photoelectron statistics in the relevant energy range for dark matter recoils. Similarly the drift velocity in liquid xenon has saturated [19] and will not be affected by $15 \%$ variations in $E_{1}$.

The amplitude of the secondary will be affected in three ways: the number of electrons escaping recombination from the interaction region, the extraction efficiency from the surface, and the photon generation in the gas phase. The first of these is inversely correlated with the decline in the primary signal. For low-energy $\gamma$-rays at $8.9 \mathrm{kV} / \mathrm{cm}$ the charge escape fraction is $\sim 0.7$, and the local slope is $\sim 0.015(\mathrm{kV} / \mathrm{cm})^{-1}[20]$. The $<15 \%$ variation in $E_{1}$ then translates into a $<4 \%$ variation in the secondary signal. For nuclear recoil signals a worse case scenario is that the secondary signal has a linear dependence on $E_{1}$ starting from the origin. Variations in $E_{1}$ then map one-to-one onto the secondary signal. If the charge signal for nuclear recoils shows an initial fast rise with a shallower slope at higher fields, as shown by [12], the variation will be somewhat lower and closer to that shown by $\gamma$-rays. At $8.9 \mathrm{kV} / \mathrm{cm}$ the extraction of the charge into the gas phase is effectively already $100 \%$ and well into the plateau region [21]. The final affect on the secondary scintillation signal will be from the electroluminescent output from the gas phase. This is known to depend linearly on $E_{\mathrm{g}}$ [22-25], but there is some scatter in the coefficients reported. A $15 \%$ variation in $E_{\mathrm{g}}$ would translate into a $12-16 \%$ variation in the electroluminescent output per unit gas drift length. This effect will be mitigated if the field variation is actually due to a variation in the distance between the liquid surface and the anode mirror, i.e the gas drift length. Finally it should be noted that the above systematic position dependent variations can, in principle be corrected for by using the position sensitivity.

The stainless-steel wire grids were strung from continuous lengths of $100 \mu \mathrm{m}$ diameter wire wound around copper formers. The position of each wire was controlled by slots machined into the formers (see Fig. 6). The wires were tensioned using two techniques. Firstly the formers were elastically deformed whilst the wire was wound and secondly the winding jig tensioned the wire as it was wound. Once the grid winding was complete the wire was anchored and the formers were then released from their restraining jigs.

Some consideration was given to whether the anode mirror should be coated to enhance its reflectivity. The performance of polished copper is quite uncertain at VUV wavelengths, depending on the surface finish, oxidation state and possible LXe condensation onto the cold surface in the gas phase. Only a single measurement has been found, indicating $R=27 \%$ for normal incidence for a clean-cut surface [26]. However the simplicity of leaving this surface as is, the uncertainty of using coatings in a high-field application and the desire not to compromise the spatial reconstruction argued for not using any coating.

\subsection{The xenon transport system}

Transfer of xenon in and out of the target vessel is independent of the cooling system. Two copper access pipes are included for movement of xenon in and out of the target vessel (see Fig. 7). One surfaces above the liquid level in an unconfined volume and is used as a 'Gas Inlet'. The second has a double-tube structure with an open-ended inner pipe connected directly to the main liquid volume, and an outer pipe which vents to the outside through the 'LXe Outlet'. The outer pipe is sealed at the top and the inner one opens above the liquid surface and essentially allows a 'syphon' action during emptying. 


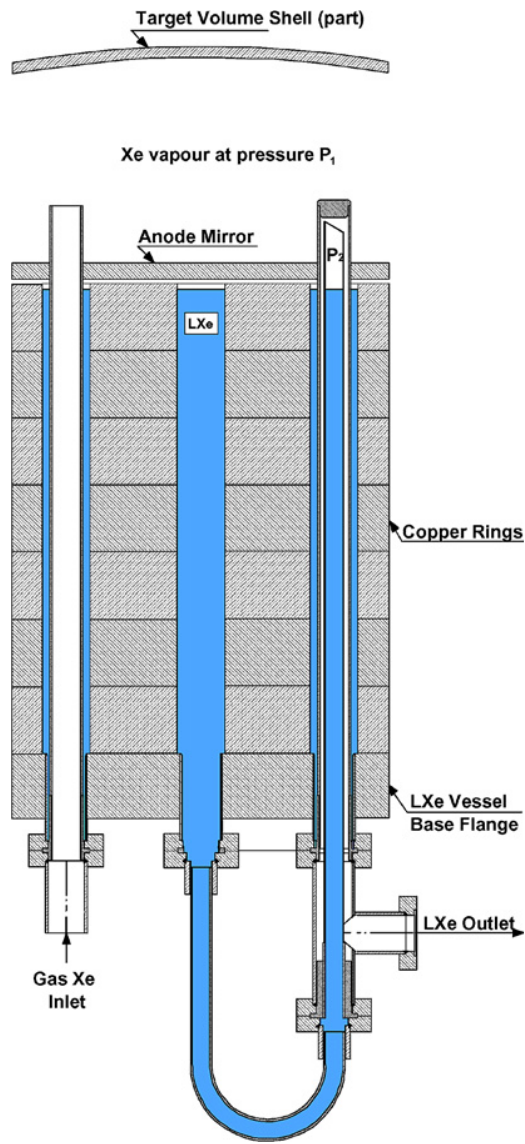

Fig. 7. Arrangement of the pipework used for xenon transfer in and out of the target vessel.

\subsection{The target vessel}

The containment vessel for the xenon target must perform as both a high vacuum vessel, for purity reasons, and a pressure vessel for safety reasons. The pressure vessel design was done following the relevant British Standard (BS5500:1997). This safety standard dictates the cylindrical wall, dome and bottom flange thicknesses which are dependent on the material and processes used. The vessel was required to be certified to 6 bar absolute. The material of choice was determined by requiring the product of total mass times radioactive content be a minimum. Added to this prime requirement was then the need for the material to be suitable for manufacture of the vessel. OFHC copper type $\mathrm{C} 103$ was selected. This required 4-mm wall thickness on the cylindrical sections, $3 \mathrm{~mm}$ for the spun domes and $25 \mathrm{~mm}$ for the flat bottom flange. To minimise the likelihood of inclusion of any impurities electron-beam welding was used throughout and the number of welds was kept to a minimum. In particular the cylindrical section was rolled in one piece. Stainless-steel parts were used for some specialist components which would have been very difficult to make out of copper, such as vacuum knife-edge flanges and vacuum HV feedthroughs for which commercial parts were used. Where necessary these stainless parts were also

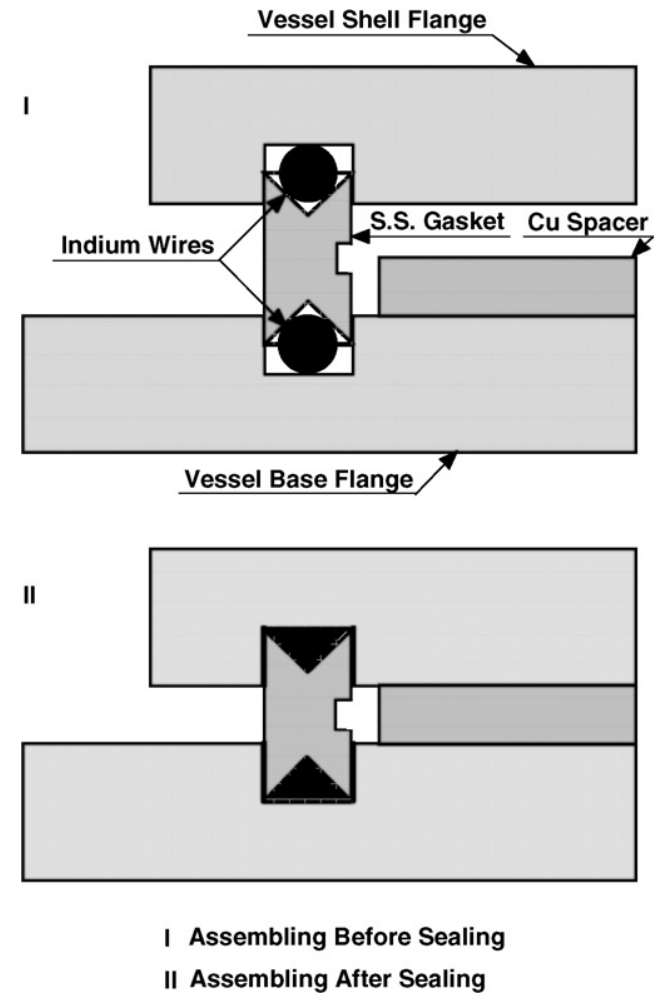

Fig. 8. Vacuum seal between the cylindrical wall section and the bottom flange before (I) and after (II) sealing.

electron-beam welded to the copper. Welding techniques adapted to our requirements were developed by The Welding Institute, UK [27], in close cooperation with us. This included setting the welding parameters and optimising the structural/thermal design of the weld joints. All safety critical welding was done by certified processes and copper witness plates were used to ensure proper and complete breakthrough as all welds were required to show full-depth penetration. Special jigging was required to hold all seams for welding securely in place during the process. On completion all joints were leak-tested down to the level of $\sim 10^{-10}$ mbar $1 \mathrm{~s}^{-1}$.

The electrical feedthroughs for the PMT dynode connections were fitted in with screw threads with indium ${ }^{2}$ coated onto them using an ultrasonic soldering iron. The demountable vacuum seal between the cylindrical section and the bottom flange was done using a stainless-steel gasket with double knife edges and an indium wire at both copper surfaces (see Fig. 8). All copper parts were cleaned, starting with a coarse hand polishing with stainless wire wool, a fine polishing with copper wire wool, an ultrasonic bath using 2\% CITRANOX [29] in de-ionised water and a

\footnotetext{
${ }^{2115} \mathrm{In}$ is a $\beta^{-}$emitter with $\beta_{\max }=482 \mathrm{keV}$. The only $\gamma$-ray line associated with this decay has a branching ratio of $\sim 10^{-6}$ [28]. Our seals contains a total of $40 \mathrm{~g}$ of indium giving an activity of $\sim 10 \mathrm{~Bq}$. However this is all located behind enough passive shielding that the low-energy $\beta$ particles cannot produce any significant interaction rate in the fiducial volume.
} 
high-pressure wash using pure water. The polishing phase was done using a powered rotation table specially built for the purpose and polishing was always applied along the line of existing machining marks.

\section{The cooling system}

Cooling is done using liquid nitrogen (LN2). The internal reservoir, located under the target vessel, holds 361 . There are two thermal links between this reservoir and the target vessel (see Fig. 9). The first link is a conduction path provided by flexible bundles of thick copper wires thermally anchored to a hollow copper cooling flange attached to the underside of the target vessel. The flexibility helps decouple acoustic/mechanical noise in the LN2 reservoir from the LXe chamber. The other end of the bundle dips into the liquid nitrogen and the thermal impedance depends on the depth of the liquid. The bundle is welded and polished at both ends for good thermal matching. A second thermal path is provided by a direct connection between the nitrogen reservoir and the hollow cooling flange. This allows cold boil-off gas to be used as additional coolant and provides the means for active thermal control with minimum cryogen usage, which is important during stand-alone operation underground.

Four external pipes are connected to the cooling system. The first is the liquid nitrogen delivery line and this terminates inside the reservoir close to the top. A second pipe also opens to the top of the reservoir, whilst the remaining pair of pipes connect to the cooling flange via the bundles. These last two pipes are fitted with control valves which regulate the internal pressure and the flow rate through the hollow cooling flange. During initial cool-down the flow through the cooling flange is increased to allow bulk liquid flow into it. Once cold, the gas flow through the cool- ing flange provides a fine temperature control mechanism whilst the copper cable bundles provide the main thermal link balancing the average heat load. The heat load is reduced by the use of thermal insulation around both the target vessel and the nitrogen chamber (see Fig. 10). The nominal operating temperature is around $-100^{\circ} \mathrm{C}$ and the heat load is, as expected, $\sim 40 \mathrm{~W}$, giving a design holdtime between refills of $\sim 2$ days.

\section{The outer vacuum jacket}

The design principles for the vacuum jacket were much the same as for the target vessel, except that the pressure rating was reduced to 4.3 bar absolute. The safety standard for pressure vessels dictated the material thicknesses and process standards and the same attention to background and cleanliness was imposed. Hence OFHC copper was used, with electron-beam welding and minimisation of the number of seams; the cylindrical section of this larger vessel was also made from just one rolled plate. The bottom flange has an included domed section and the vacuum seals were all done in the same way as for the target vessel.

\section{The gas purification system and safety reservoirs}

Fig. 11 shows a schematic of the xenon gas purification system. The main requirement is to be able to remove electronegative contaminants which will prevent the ionisation electron drift and suppress the secondary signal. For ZEPLIN-III the maximum drift time is $\sim 17 \mu$ s. To avoid significant attachment within this time typically requires liquid xenon purity below the parts per billion level for these impurities; this is beyond that available through commercial purchase. In addition the level of radioactive krypton needs to be kept as low as possible as the $\beta$-decay of

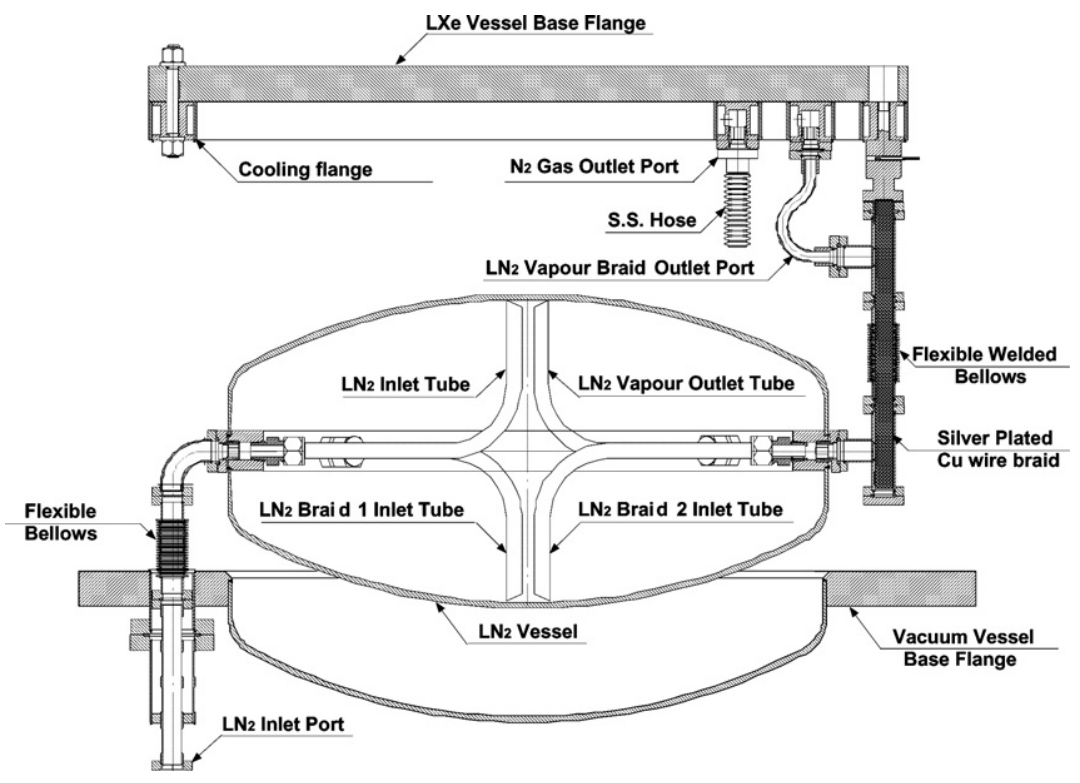

Fig. 9. Thermal control system elements. 


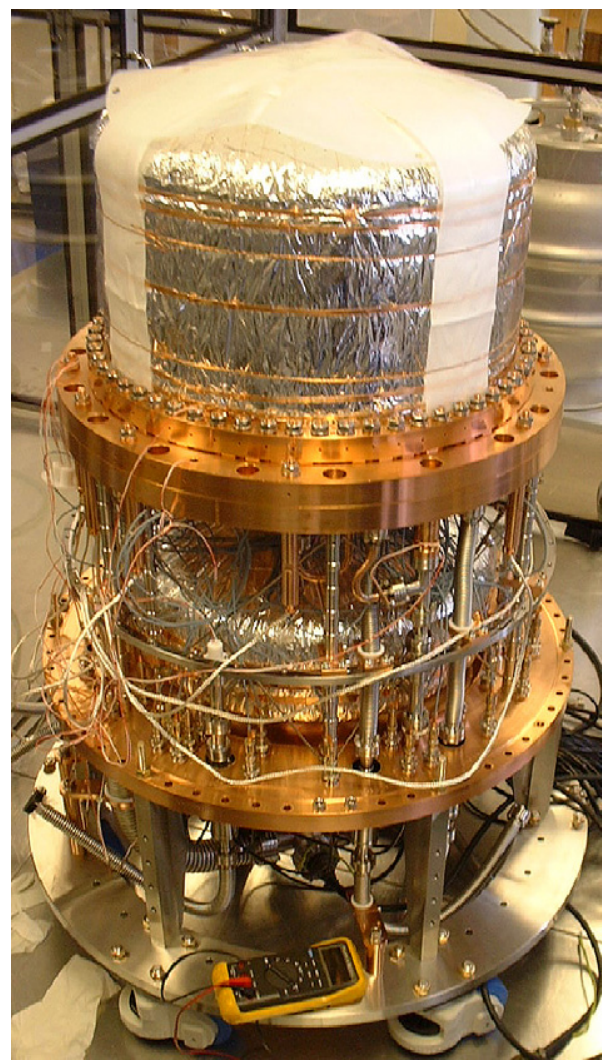

Fig. 10. The assembled instrument without its vacuum jacket giving a view of the target vessel (top) and liquid nitrogen reservoir both covered with thermal insulation.
${ }^{85} \mathrm{Kr}$ gives a continuum energy deposit down into the level expected from elastic scattering of WIMPs. Although our drift time requirement is modest compared with the millisecond lifetimes already achieved by others $[30,14]$ it still requires a significant purification activity. An all-metal bakeable gas system has been used. The system is pumped by a combination of oil-free scroll and turbo-molecular pumps. The xenon gas is contained in two large stainlesssteel cylinders fitted with high purity all-metal UHV valves and regulators. These two cylinders stand in cooling jackets allowing them to be cooled to liquid nitrogen temperatures. Two SAES [31] getters are used. Fine particle filters $(0.5 \mu \mathrm{m})$ are fitted to all gas delivery lines. The gas system is fitted with a mass spectrometer which is used both for helium leak testing and residual gas analysis. The base vacuum attainable in the system is $\sim 10^{-8} \mathrm{mbar}$, dominated by $\mathrm{H}_{2}$; a partial $\mathrm{H}_{2} \mathrm{O}$ pressure of $\sim 10^{-10}$ mbar was achieved prior to the xenon input. The detector itself is connected without valves to a port on the main volume of the gas purification system. Another port is connected to the large volume safety reservoirs with only a burst disk between them. This is not only to guard against the safety risk associated with catastrophic failure of the target vessel under overpressure, but also to avoid loss of xenon. The two gas cylinders contain $50 \mathrm{~kg}$ of xenon supplied by ITEP from stock collected from underground sources between 20 and 40 years ago. This xenon has a very low radioactive krypton content $(5 \mathrm{ppb} \mathrm{Kr})$, and the contribution to the low-energy background due to the ${ }^{85} \mathrm{Kr} \beta^{-}$decay is about

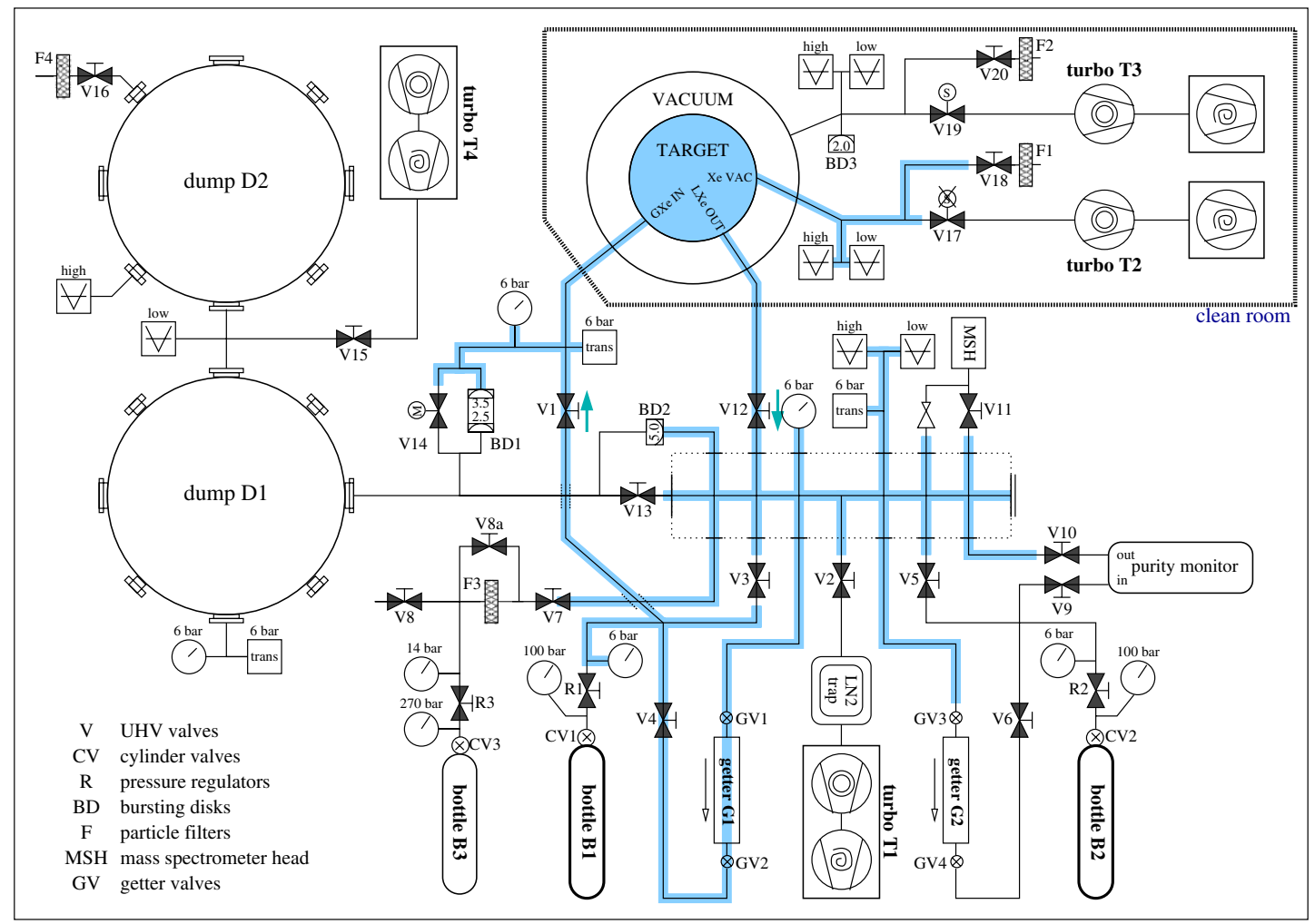

Fig. 11. Schematic diagram of the gas purification system. 
$1 \%$ of the background from the current PMTs [11] and hence will not be a significant problem even after the lower background PMTs are installed. A final component of our gas purification system is a novel portable chamber for electron lifetime measurements which will be described elsewhere [32].

\section{The data acquisition system}

The 31 PMT signals are fed into wideband amplifiers and split into a dual dynamic range data acquisition system (DAQ). This ensures sensitivity to very small primary scintillation signals containing only a few photoelectrons (phe) as well as to large secondaries without saturation. All 62 channels are sampled at $500 \mathrm{MS} / \mathrm{s}$ by 8-bit ACQIRIS digitisers. For the collection of 'dark matter' data a PMT gain of $2 \times 10^{5}$ will be used. Such a low gain should avoid internal PMT saturation effects following very large secondary scintillation signals. Wideband amplifiers add electronic gain in two stages. The first stage is $(\times 10)$ with a noise referred to the input of $30 \mu \mathrm{V}$ rms. They then feed into adjustable attenuators which are used to equalise the single photoelectron response for each PMT. The outputs from this stage then feed into the 31 low-gain digitisers as well as into the next stage $\times 10$ wideband amplifiers. The highand low-gain input channels thus have a factor of 10 gain difference which can be further expanded by adjusting the full-scale ranges on the digitisers. A simple threshold trigger signal is derived from a summing amplifier, with inputs from all PMTs, fed into a discriminator whose output provides an external trigger for the ACQIRIS digitisers. This trigger cannot differentiate between primary and secondary scintillation signals. A more sophisticated trigger using a time to amplitude converter can provide a width measure and differentiate the very short primary scintillation signals ( $\sim 30$ ns time-constant) from the much more extended secondary scintillation signals ( $\sim 1 \mu$ s duration). The maximum delay between primary and secondary scintillation signals in ZEPLIN-III is $\sim 17 \mu \mathrm{s}$. A LINUX-based software application reads out the digitiser crates. A FIFO-type memory buffer, accessed independently by two CPUs for data transfer and write-out, reduces the overall dead time. An acquisition rate of 100 events/s can be sustained.

\section{Commissioning cool-down tests}

The first cool-down test was designed to verify the thermal control system and to test out the PMT array. For this test the anode and cathode electrodes were replaced by a copper plate located just $8 \mathrm{~mm}$ above the PMT array. Thirty-one ${ }^{241} \mathrm{Am}$ radioactive sources were vacuum-sealed into this plate with a thin copper foil overlay to prevent leakage of radioactivity and to stop $\alpha$-particles from interacting in the xenon. These then provided a source of low-energy (mainly $59.6 \mathrm{keV}$ ) photons. For subsequent cool-down tests the radioactive sources had been removed and the full electric field system installed in its final configuration.

\subsection{Cooling system}

The cooling system performance during the first cooldown was as expected. The initial cool-down period used 2001 of liquid nitrogen and progressed at $\sim 5^{\circ} \mathrm{C} / \mathrm{h}$. An array of temperature sensors was used to monitor critical points within the instrument. One of these, on the lower face of the cooling flange on the bottom of the target, is used as the control temperature and its reading is compared with a set temperature in the controller to automatically operate two valves: one which exhausts straight from the gas volume of the nitrogen reservoir, and one which exhausts through the cooling ring. Once down at the nominal operating temperature $\left(\sim-100^{\circ} \mathrm{C}\right)$ the temperature of the target vessel is stable to better than $0.2{ }^{\circ} \mathrm{C}$ and the liquid nitrogen usage drops to $\sim 201 /$ day as expected. Fig. 12 shows some key house-keeping parameters monitored over a $24 \mathrm{~h}$ period during the second cool-down test. The upper trace is from the temperature sensor on the cooling flange and the periodic behaviour is due to the control system. The lower trace is then the temperature of the base plate of the target vessel itself.

\subsection{PMT array}

Pulse height spectra, pulse waveforms and single photoelectron spectra were collected from all PMTs during the first cold-run both with the DAQ electronics just described and with a pulse height analysis (PHA) set-up using a multichannel analyser (MCA). These confirmed correct operation of all 31 PMTs in the array, including 1000

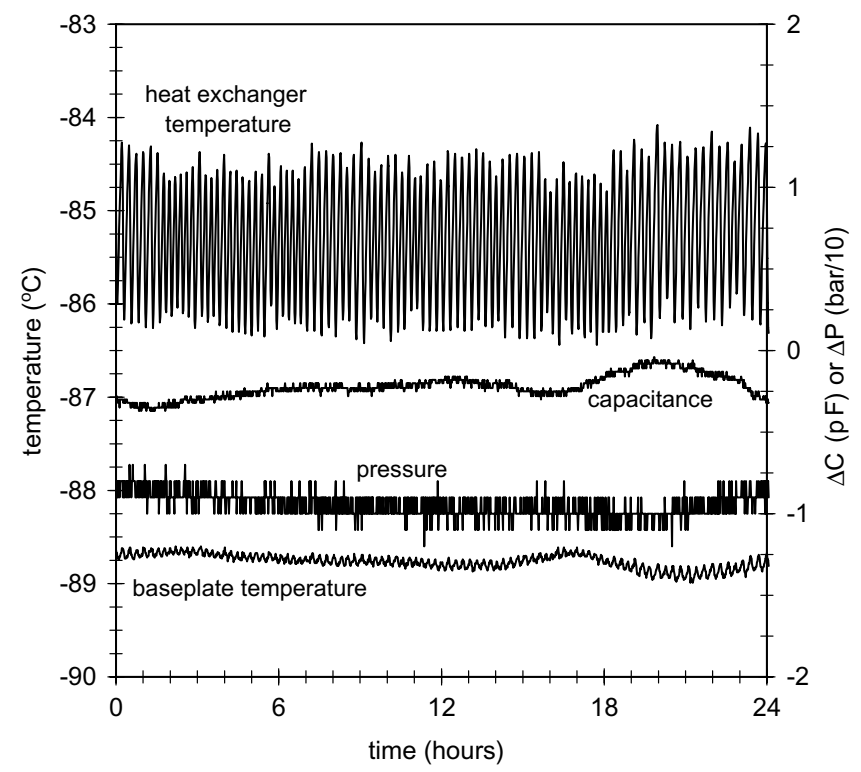

Fig. 12. Some key system house-keeping parameters monitored over a $24 \mathrm{~h}$ period. 

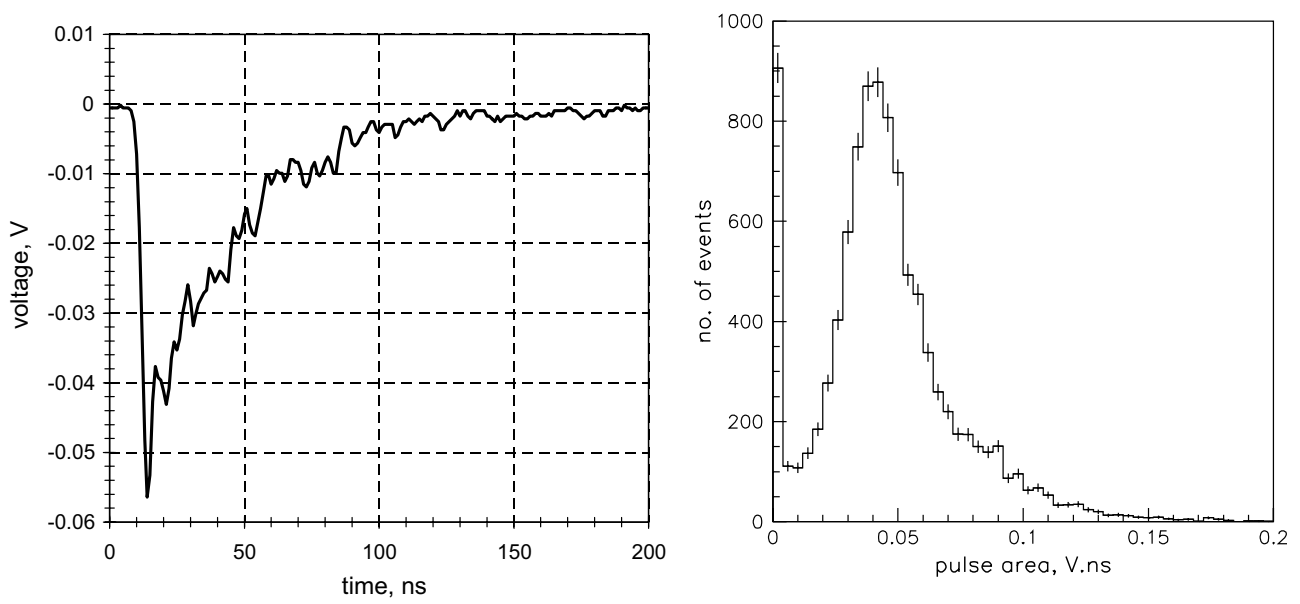

Fig. 13. A primary scintillation pulse from a low-energy $\gamma$-ray interaction and a single electron response from one of the PMTs. Both were obtained with $-2 \mathrm{kV}$ on the cathode; however there was an additional $\times 10$ amplifier present for the single electron measurement.

crimped connections! A primary scintillation pulse from a low-energy $\gamma$-ray emitted by an ${ }^{241} \mathrm{Am}$ source is shown in Fig. 13. This shows the characteristic decay time of $\sim 40$ ns. The single electron spectra show well resolved peaks and these were used to set the amplifier gains in order to normalise all channels to the same overall gain.

\subsection{Scintillator performance}

LXe scintillates in the vacuum ultraviolet (VUV), near $175 \mathrm{~nm}$, with a yield comparable to the best scintillator crystals. The VUV luminescence is produced by the decay of singlet and triplet states of the $\mathrm{Xe}_{2}^{*}$ excimer. These can be formed directly by excited atoms left by the interacting particle or as a result of recombination into an excited state along the particle track $[16,33]$.

Fig. 14 shows typical MCA spectra taken from one PMT when the internal ${ }^{241} \mathrm{Am}$ sources were in place. The bottom spectrum was taken with the whole arrangement covered with liquid xenon. The two spectral features are the $59.6 \mathrm{keV}$ line from ${ }^{241} \mathrm{Am}$ and a blend of the $26.3 \mathrm{keV}$ ${ }^{241} \mathrm{Am} \gamma$-ray with a $30 \mathrm{keV}$ line resulting from escape of $\mathrm{Xe} \mathrm{K}$-shell fluorescence photons. Using the MCA single photoelectron spectrum from this PMT gives a signal level of $\sim 12 \mathrm{phe} / \mathrm{keV}$. For this measurement there is no applied electric field. The top spectrum was taken with the liquid level between the source and the PMT window. The interactions occurred mainly in the liquid phase and the improved light collection (up to $\sim 17 \mathrm{phe} / \mathrm{keV}$ ) is a result of total internal reflection at the liquid gas interface due to the refractive index mismatch. The resolution from the two-phase spectrum is $\sim 13 \%$ FWHM.

\subsection{Two-phase operation}

Once the radioactive sources used for the measurements in the previous section had been removed the second and subsequent cold-runs have successfully loaded the detector

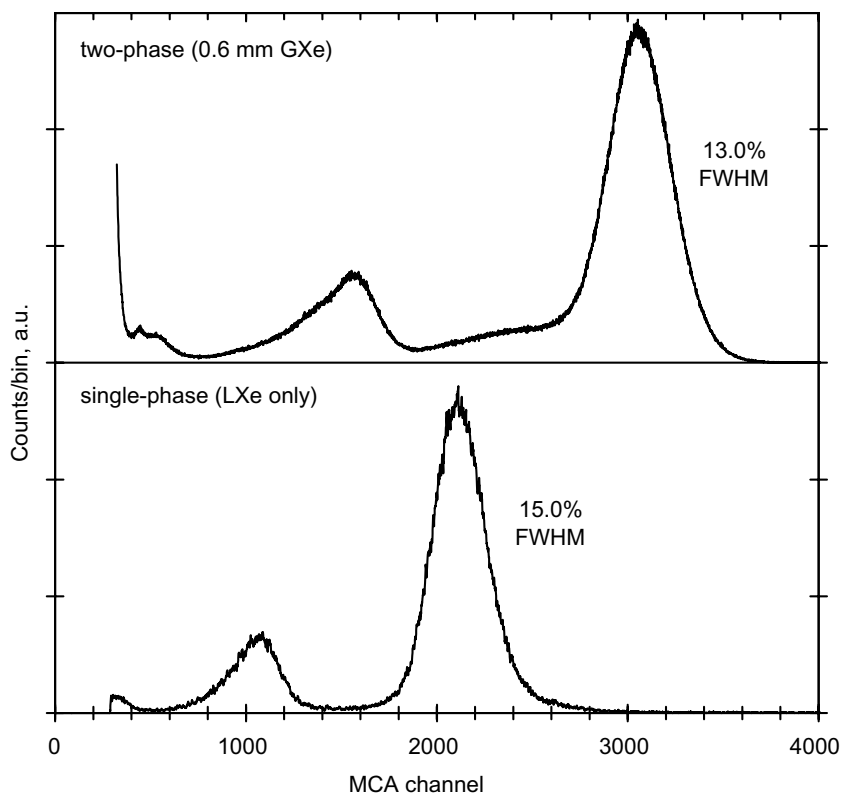

Fig. 14. Typical MCA scintillation spectra obtained with the temporary ${ }^{241} \mathrm{Am}$ sources located a few $\mathrm{mm}$ above the PMT array. The two panels correspond to two different liquid xenon levels. The highest energy peaks in both spectra are centred at $59.6 \mathrm{keV}$.

with liquid xenon. A capacitive level-sensing system probes the liquid xenon height with sub-mm accuracy at three locations in the chamber. A signal from one of these coaxial capacitor structures, readout to $\pm 0.03 \mathrm{pF}$, is shown in Fig. 12. In underground operation, these sensors will be integrated with an active levelling system in order to maintain the electrodes parallel to the liquid surface, guarding the heavily shielded detector against any structural deformation of the underground cavern.

With the xenon filled to its nominal depth, but with no applied electric field, ${ }^{57} \mathrm{Co} \gamma$-ray spectra were recorded with an uncollimated source located above the detector. A photoelectron yield of $5 \mathrm{phe} / \mathrm{keV}$ was obtained for the centre of the chamber, with a FWHM $\simeq 25 \%$. Such a high yield 
exceeds that predicted by Monte Carlo simulations (3.4$4.0 \mathrm{phe} / \mathrm{keV}$ ) [11]. A higher LXe scintillation yield than considered in those simulations (60 photons $/ \mathrm{keV}$, $W_{\mathrm{ph}}=16.7 \mathrm{eV}$ ) could explain this difference. A corresponding decrease in energy threshold, a key parameter of WIMP detectors, is expected.

After the zero-field tests, $13.5 \mathrm{kV}$ were applied between the cathode grid and the anode mirror, setting up a field of $3.0 \mathrm{kV} / \mathrm{cm}$ in the liquid. In our surface laboratory, with its high background, it is not advisable to apply fields in excess of these values due to potential damage to the PMTs from such high photon fluxes. Fig. 15 shows a typical signal from a $\gamma$-ray interaction in the LXe. The fast scintillation is the primary signal, S1, caused by direct excitation created by the photoelectron. The second, broader signal, $\mathrm{S} 2$, occurs when the ionisation released at the interaction site has drifted to the liquid surface and has been extracted into the gas phase. Once in the gas phase the electric field is strong enough to cause excitation leading to a burst of additional photons. The time delay depends on the depth at which the interaction happened and the drift velocity at our operating fields $(\sim 2.5 \mathrm{~mm} / \mu \mathrm{s})$. The width of the secondary depends on the gas gap and the electric field in the gas. The secondary emission levels off as the charge drifts across the gap. The rise and fall times are due to a combination of extraction dynamics, charge diffusion and the gas scintillation time-constant.

Further ${ }^{57} \mathrm{Co}$ measurements were made at this field and the result is shown in Fig. 16 for the ionisation (S2) and scintillation (S1) channels. Two spectra are shown in each panel, both reconstructed using all PMTs, with individual gains and QEs equalised. The shaded one, however, only includes events in which the peak signal occurred in one of the inner 7 PMTs. This 'collimated' spectrum shows an S1 light yield of $1.8 \mathrm{phe} / \mathrm{keV}$, approximately $35 \%$ of

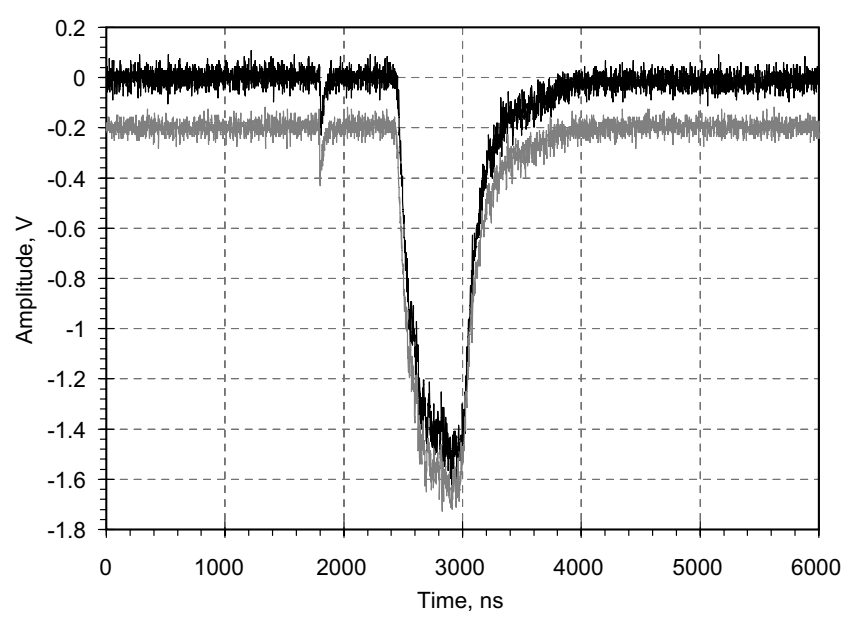

Fig. 15. Summed waveforms from a $\gamma$-ray event showing a fast primary pulse followed by the secondary wider pulse from electroluminescence in the gas phase caused by ionisation drifted from the event site. The two traces shown are from the dual range DAQ. The low-sensitivity data have been multiplied by 10 .
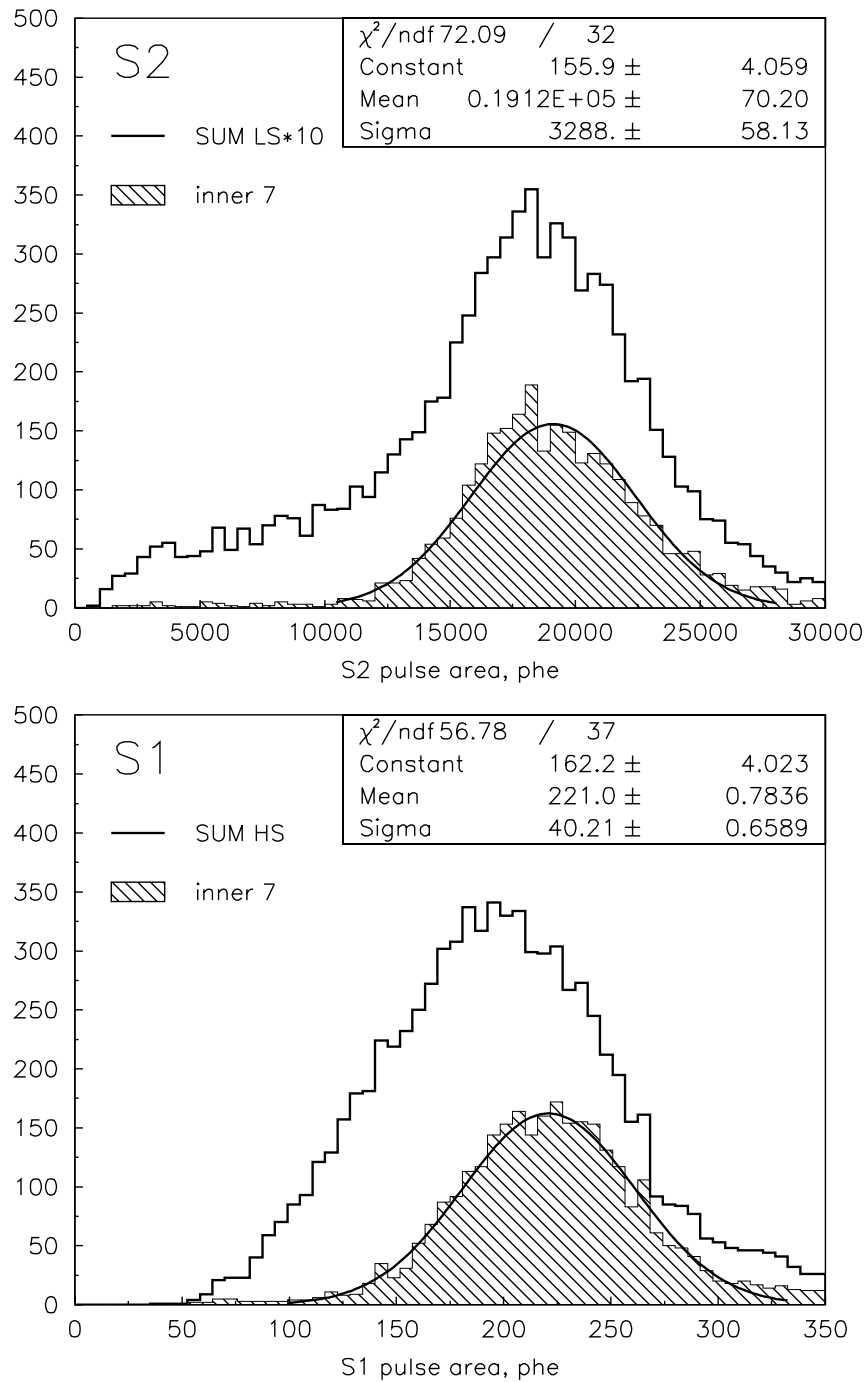

Fig. 16. Pulse area spectra for S2 (top) and S1 (bottom) obtained with an uncollimated ${ }^{57} \mathrm{Co}$ source placed above the instrument, for an electric field of $3.0 \mathrm{kV} / \mathrm{cm}$ in the liquid. The peak corresponds to the blended $122.1 \mathrm{keV}$ and $136.5 \mathrm{keV}{ }^{57} \mathrm{Co} \gamma$-ray lines $(85.6 \%$ and $10.7 \%$ relative intensities, respectively).

the zero-field value. A $F W H M \simeq 40 \%$, calculated using a single-Gaussian fit to the $122.1 \mathrm{keV}$ and $136.5 \mathrm{keV}{ }^{57} \mathrm{Co}$ $\gamma$-ray lines, is consistent with the degradation of the zerofield energy resolution due to photoelectron statistics. The $\mathrm{S} 2 / \mathrm{S} 1$ ratio is $\simeq 100$ as expected at this field. The broad shoulder on the low side for the uncollimated spectra are purely due to light collection variations towards the edge of the xenon volume. Most of these fall outside the fiducial volume and the remainder can be corrected using 3-D position reconstruction information.

One of the key design drivers of ZEPLIN-III was the ability to resolve each interaction point in the three dimensions. A position reconstruction algorithm was developed from simulated datasets which will provide sub-cm resolution in the horizontal plane [34]. Even before this is applied to real data, this spatial sensitivity is well demonstrated in Figs. 17 and 18, showing an event in which two interactions 


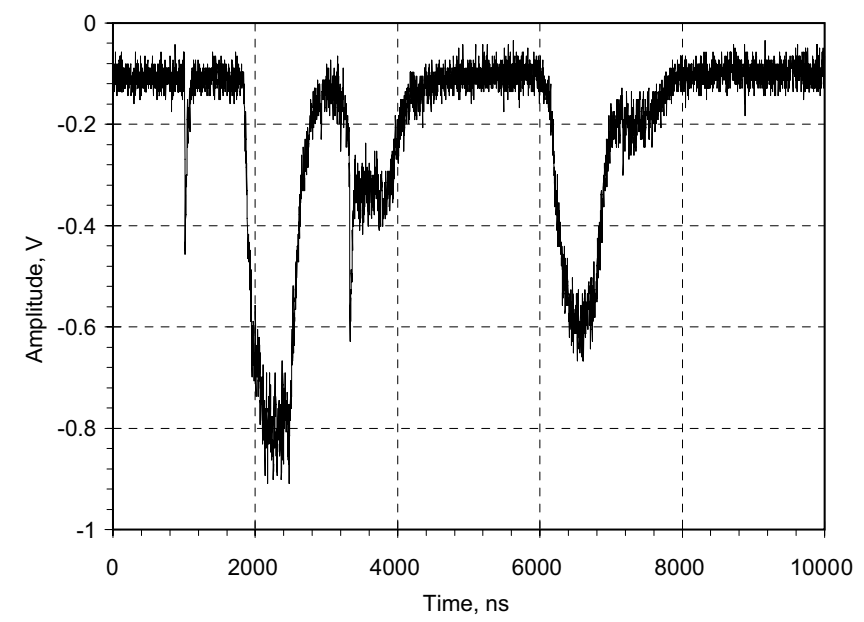

Fig. 17. Summed waveforms from two overlapping $\gamma$-ray events both showing fast primary signals followed by secondary signals.

have overlapped in time. Moreover there are at least four secondary signals. Without position sensitivity it would not be possible to separate these two events just from the summed signals. However, looking at the individual PMT traces (left-hand panel in Fig. 18) it is immediately obvious that these two events have happened in very different parts of the detector (right-hand panel) and they can be unambiguously separated. They are both double-Compton scatters.

Ultimately, the performance of a dark matter detector is judged from its ability to discriminate between nuclear and electron-recoil interactions, the latter dominating the background of a shielded instrument operating underground. Demonstration of this key feature is given in Fig. 19, which shows how the S2/S1 ratio varies with energy when a

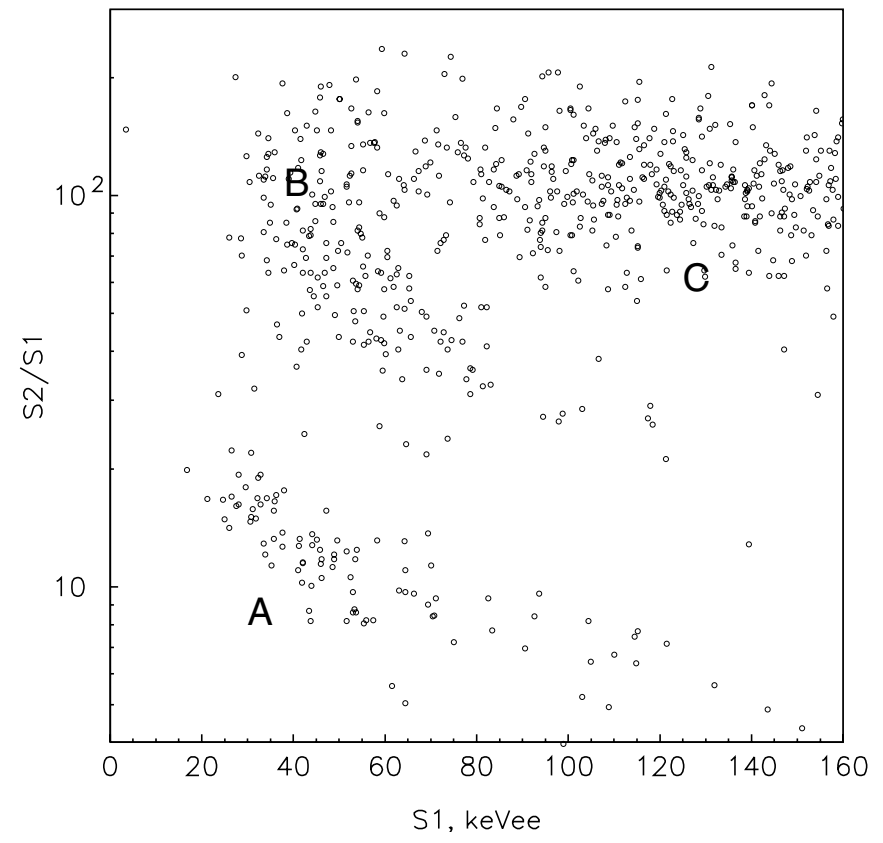

Fig. 19. Scatter plot of the $\mathrm{S} 2 / \mathrm{S} 1$ ratio as a function of electron-equivalent energy $(\propto S 1)$ for single-scatter events obtained with an Am-Be source located above the detector. For elastic nuclear recoil events (population A) the energy scale must be multiplied by 1.95 , which comes from the combination of the inverse quenching factor, $(1 / 0.19[35,17,36])$ and the relative $\mathrm{S} 1$ suppression factor at our operating field $(\sim 0.37)$. Population $\mathrm{B}$ corresponds to the de-excitation of the $40 \mathrm{keV}^{129} \mathrm{Xe}$ inelastic level (plus some nuclear recoil energy). Population $\mathrm{C}$ is the $\gamma$-ray population, also associated with the source.

$10 \mathrm{mCi} \mathrm{Am}-\mathrm{Be}(\alpha, \mathrm{n})$ source is placed above the detector. The field within the liquid was maintained at $3 \mathrm{kV} / \mathrm{cm}$ as before. Discrimination between elastic recoils (population A) and $\gamma$-ray interactions (C) is clearly demonstrated.
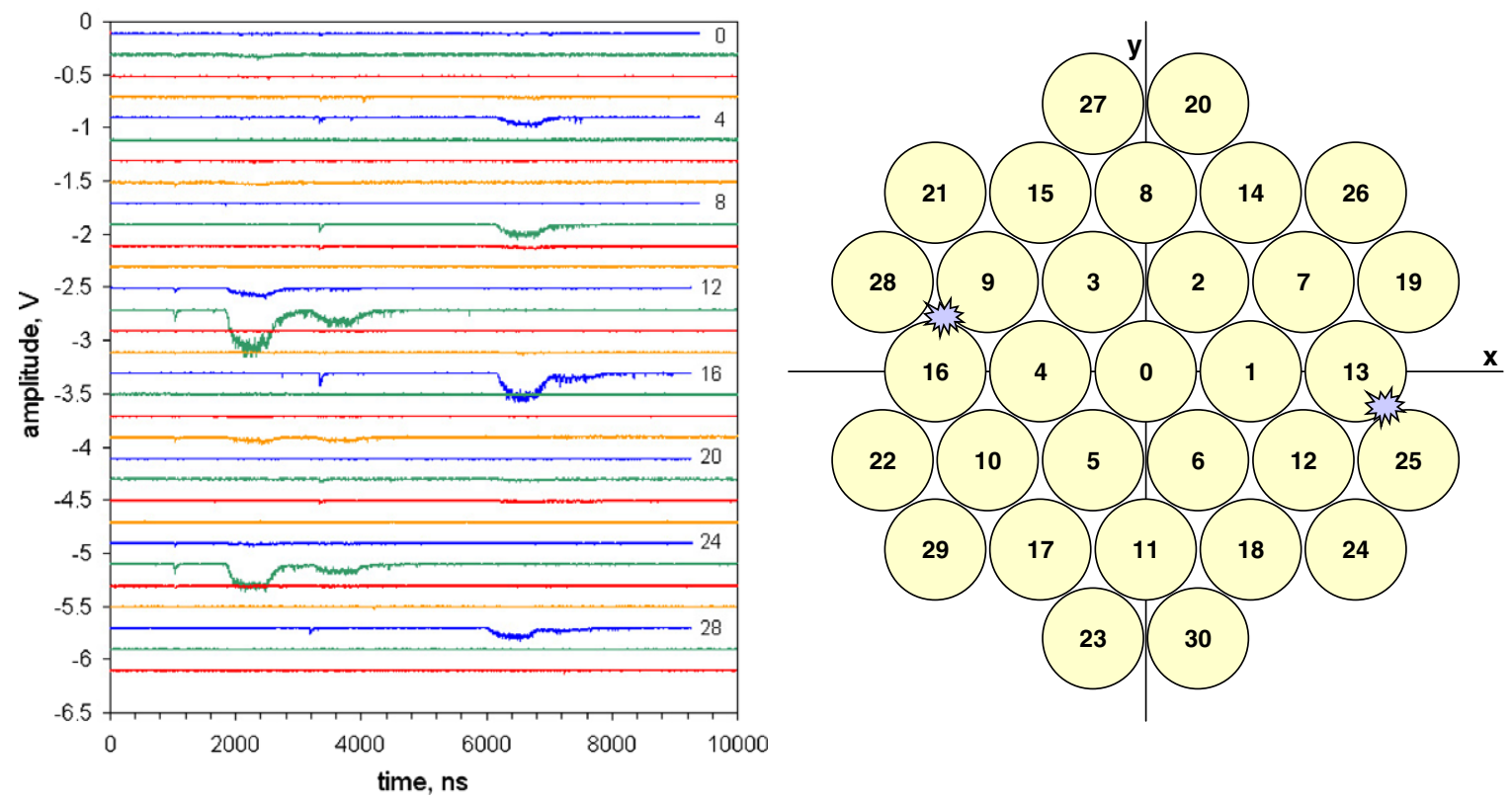

Fig. 18. Individual waveforms from the 31 PMTs showing how the two overlapping events can be separated using position reconstruction. The reconstructed positions are indicated on the right-hand panel and the size of the symbols is representative of the position resolution. 
Population B corresponds to neutron inelastic scattering off ${ }^{129} \mathrm{Xe}$ nuclei; in this instance some (quenched) nuclear recoil energy is followed by emission of a $40 \mathrm{keV} \gamma$-ray from nuclear de-excitation.

\section{Summary}

The key design features of the ZEPLIN-III instrument have been described. The challenging and pioneering aspects of the manufacturing technologies and procedures have been detailed and first commissioning data have been presented to demonstrate the successful completion of build of this instrument. The commissioning data have verified the high light collection, the 3-D position sensitivity and the ability to separate nuclear recoils from electron recoils. Fuller characterisation of ZEPLIN-III as a dark matter detector, in particular establishing its internal radioactive level and its discrimination factor, will be done underground in a lower background environment.

\section{Acknowledgements}

This work has been funded by the UK Particle Physics and Astronomy Research Council (PPARC). We would like to acknowledge the superb copper machining achieved within the Imperial College Physics Department workshop led by R. Swain, and the development of new welding techniques by The Welding Institute.

\section{References}

[1] T.J. Sumner, New Astron. Rev. 49 (2005) 277-281.

[2] H.M. Araújo, in: Proc. Int. Conf. Dielectric Liquids, Coimbra, Portugal, IEEE Press, 2005, p. 305 (IEEE Cat. 05CH37643).

[3] G.J. Alner et al., New Astron. Rev. 49 (2005) 259-263.
[4] G.J. Alner et al., Nucl. Instrum. Meth. A 555 (2005) 173-183.

[5] B.A. Dolgoshein, V.N. Lebedenko, B.U. Rodionov, JETP Lett. 11 (1970) 513.

[6] T.J. Sumner et al., in: D. Kieda, M. Salamon, B. Dingus (Eds.), Proc. 26th Int. Cosmic Ray Conf., vol. 2, 1999, p. 516.

[7] A.S. Howard et al., in: N.J.C. Spooner, V. Kudryavtsev (Eds.), Proc. 3rd Int. Workshop on the Identification of Dark Matter, World Scientific, Singapore, 2001, p. 457.

[8] D.Yu. Akimov et al., in: N.J.C. Spooner, V. Kudryavtsev (Eds.), Proc. 4th Int. Workshop on the Identification of Dark Matter, World Scientific, Singapore, 2003, p. 371.

[9] D. Davidge, PhD Thesis, University of London, 2003.

[10] J. Dawson, PhD Thesis, University of London, 2003.

[11] H.M. Araújo et al., Astropart. Phys. 26 (2) (2006) 140-153.

[12] E. Aprile et al., Phys. Rev. Lett. 97 (2006) 081302.

[13] H.M. Araújo et al., Nucl. Instrum. Meth. A 521 (2004) 407-415.

[14] N.J.T. Smith et al., in preparation.

[15] ANSYS Inc. <www.ansys.com>.

[16] S. Kubota et al., Phys. Rev. B 20 (8) (1979) 3486-3496.

[17] E. Aprile et al., Phys. Rev. D 72 (2005) 072006.

[18] J. Dawson et al., Nucl. Instrum. Meth. A 545 (2005) 690-698.

[19] L.S. Miller et al., Phys. Rev. 166 (3) (1968) 871-878.

[20] T.Ya. Voronova et al., Sov. Phys. Tech. Phys. 34 (7) (1989) 825827.

[21] E.M. Gushchin et al., Sov. Phys. JETP 49 (5) (1989) 856-858.

[22] H.N. Ngoc et al., Nucl. Instrum. Meth. A 158 (1979) 128.

[23] A. Parsons et al., IEEE Trans. Nucl. Sci. 36 (1) (1989) 931-935.

[24] D.Yu. Akimov et al., Phys. Atom. Nucl. 61 (7) (1998) 1241-1244.

[25] A.C. Fonseca et al., IEEE Trans. Nucl. Sci., in press.

[26] D.R. Lide, CRC Handbook of Chemistry \& Physics, 86th ed., 2005, pp. 12-119.

[27] http://www.twi.co.uk/.

[28] C.M. Cattadori et al., Nucl. Phys. A 748 (2005) 333-347.

[29] http://www.alconox.com.

[30] M. Ichigea et al., Nucl. Instrum. Meth. A 333 (1993) 355-363.

[31] http://www.saesgetters.com/.

[32] R. Walker et al., in preparation.

[33] A. Hitachi et al., Phys. Rev. B 27 (9) (1979) 5279-5285.

[34] A. Lindote et al., Nucl. Instrum. Meth. A, in press.

[35] D. Akimov et al., Phys. Lett. B 524 (2002) 245-251.

[36] V. Chepel et al., Astropart. Phys. 26 (1) (2006) 58-63. 